\title{
Analysis of pairwise correlations in multi-parametric PET/MR data for biological tumor characterization and treatment individualization strategies
}

\author{
Sara Leibfarth ${ }^{1}$ - Urban Simoncic ${ }^{1,4,5}$ • David Mönnich ${ }^{1}$ - Stefan Welz . $^{2}$ \\ Holger Schmidt ${ }^{3} \cdot$ Nina Schwenzer $^{3} \cdot$ Daniel Zips $^{2}$ - Daniela Thorwarth ${ }^{1}$
}

Received: 22 July 2015 / Accepted: 5 January 2016 / Published online: 13 February 2016

(C) The Author(s) 2016. This article is published with open access at Springerlink.com

\begin{abstract}
Purpose The aim of this pilot study was to explore simultaneous functional PET/MR for biological characterization of tumors and potential future treatment adaptations. To investigate the extent of complementarity between different PET/MR-based functional datasets, a pairwise correlation analysis was performed.

Methods Functional datasets of $N=15$ head and neck (HN) cancer patients were evaluated. For patients of group A $(N=7)$, combined PET/MR datasets including FDG-PET and ADC maps were available. Patients of group B $(N=8)$ had FMISO-PET, DCE-MRI and ADC maps from combined PET/MRI, an additional dynamic FMISO-PET/CT acquired directly after FMISO tracer injection as well as an FDG-PET/CT acquired a few days earlier. From DCE-MR,
\end{abstract}

Electronic supplementary material The online version of this article (doi:10.1007/s00259-016-3307-7) contains supplementary material, which is available to authorized users.

Sara Leibfarth

sara.leibfarth@med.uni-tuebingen.de

1 Section for Biomedical Physics, Department of Radiation Oncology, University Hospital Tübingen, Tübingen, Germany

2 Department of Radiation Oncology, University Hospital Tübingen, Tübingen, Germany

3 Department of Diagnostic and Interventional Radiology, University Hospital Tübingen, Tübingen, Germany

4 Faculty of Mathematics and Physics, University of Ljubljana, Ljubljana, Slovenia

5 Jozef Stefan Institute, Ljubljana, Slovenia parameter maps $K^{\text {trans }}, v_{\mathrm{e}}$ and $v_{\mathrm{p}}$ were obtained with the extended Tofts model. Moreover, parameter maps of mean DCE enhancement, $\overline{\Delta S}_{\text {DCE }}$, and mean FMISO signal 0-4 $\min$ p.i., $\bar{A}_{\text {FMISO }}$, were derived. Pairwise correlations were quantified using the Spearman correlation coefficient $(r)$ on both a voxel and a regional level within the gross tumor volume.

Results Between some pairs of functional imaging modalities moderate correlations were observed with respect to the median over all patient datasets, whereas distinct correlations were only present on an individual basis. Highest inter-modality median correlations on the voxel level were obtained for FDG/FMISO $(r=0.56), \mathrm{FDG} / \bar{A}_{\mathrm{FMISO}}$ $(r=0.55), \bar{A}_{\mathrm{FMISO}} / \overline{\Delta S}_{\mathrm{DCE}}(r=0.46)$, and FDG/ADC $(r=-0.39)$. Correlations on the regional level showed comparable results.

Conclusion The results of this study suggest that the examined functional datasets provide complementary information. However, only pairwise correlations were examined, and correlations could still exist between combinations of three or more datasets. These results might contribute to the future design of individually adapted treatment approaches based on multiparametric functional imaging.

Keywords Integrated PET/MR · Head and neck cancer . Treatment individualization - Multiparametric functional imaging $\cdot$ Correlation analysis $\cdot$ Hypoxia imaging

\section{Introduction}

Biological tumor characterization based on functional and molecular imaging might be highly valuable for radiotherapy (RT). On the one hand, it could allow for an improved target volume definition and an individualized dose 
prescription within the tumor according to local biological characteristics. Such dose painting strategies can be readily applied with the technical availability of intensity modulated RT (IMRT). Moreover, functional imaging might be of high value for early response assessment and potential treatment adaptation in the course of fractionated RT $[1,2]$. Other fields of application are the assessment of chemotherapy and the application of targeted agents, such as hypoxia-sensitizing or antiangiogenic drugs [3, 4].

Both positron emission tomography (PET) and magnetic resonance imaging (MRI) may provide functional information beneficial for personalized treatment strategies. PET imaging using $\left[{ }^{18} \mathrm{~F}\right]$-fluorodeoxyglucose (FDG) can be used to monitor glucose metabolism, whereas the hypoxic status of the tumor can be assessed using dedicated tracers such as $\left[{ }^{18} \mathrm{~F}\right]$-fluoromisonidazole (FMISO). Diffusion weighted MRI (DW-MRI) provides the possibility to quantify the diffusion of water molecules, which is related to cellular density [5]. Dynamic contrast-enhanced MRI (DCE-MRI) yields a temporally varying signal due to the distribution of contrast agent in blood pool and tissue. By compartmental modeling estimates of quantitative physiological parameters can be derived [6].

With the advent of combined PET/MR imaging [7, 8] the acquisition of simultaneous, intrinsically registered PET and MR data has become possible. This facilitates the comparison and combined analysis of PET- and MR-derived functional imaging data. Simultaneous PET/MR may thus be of high potential for treatment individualization $[9,10]$.

Recent studies have associated different functional imaging information with RT outcome for head and neck (HN) cancer. This applies to FDG-PET [11, 12], static as well as dynamic FMISO-PET [13-16], apparent diffusion coefficients (ADCs) inferred by DW-MRI [17], as well as DCE-MRI $[18,19]$. These studies provide a rationale to adapt RT treatment plans according to functional imaging information.

It is not clear yet if datasets from different functional imaging modalities are completely complementary, or if information is to some extent redundant. Initial analyses of correlations between different functional datasets have already been performed in recent studies. The studies of Rajendran et al. [20] and Thorwarth et al. [21] revealed good voxel-by-voxel correlation of FDG and FMISO in some HN tumors, whereas others showed no clear correlation. The biological basis of the observed correlations may be the hypoxia-inducible factor $1 \alpha$ (HIF $1 \alpha)$ [20]. Similar results were obtained by Zegers et al. [22] comparing uptake of FDG and the hypoxia PET tracer $\left[{ }^{18} \mathrm{~F}\right]-\mathrm{HX} 4$ in patients with non-small cell lung cancer. Houweling et al. [23] quantified correlations between FDG and ADC maps of HN tumors on a voxel level, and found a negative correlation in most patients. Both Newbold et al. [24] and Donaldson et al. [25] found correlations between hypoxia derived from pimonidazole staining and DCE-derived parameter maps on a region-of-interest (ROI) level. A study by Jansen et al. [26] found that neck nodal metastases with positive FMISO uptake differed significantly in median $K^{\text {trans }}$ values from those with no FMISO uptake.

Earlier studies have shown that a dynamic imaging protocol may be superior compared to a single time frame for hypoxia quantification using FMISO-PET [16]. However, in addition to a late static scan several hours post injection (p.i.), such a dynamic protocol requires a PET acquisition during tracer wash-in in the first minutes p.i. [27], which may hamper its usage in clinical routine. A positive correlation result between early FMISO and DCE information would potentially provide the possibility to infer early FMISO information from DCE, which would facilitate its clinical usage.

To address the question if available functional information of PET/MR is complementary or to some extend redundant, this study extends beyond existing studies by considering a comprehensive set of functional data. Correlations of various functional datasets are quantified on a voxel as well as on a regional level within $\mathrm{HN}$ tumors by means of the Spearman correlation coefficient. For the analysis, FDGPET, FMISO-PET acquired in the wash-in, as well as in the retention phase, ADC maps extracted from DW-MRI, and DCE-MRI derived maps are taken into account. The study is a first explorative, hypothesis generating approach to investigate the utilization of integrated PET/MR for personalized treatment strategies.

\section{Material and methods}

\section{Patient data}

Datasets from $15 \mathrm{HN}$ cancer patients from two different studies were available in total, examined with combined PET/MR (Biograph mMR, Siemens Healthcare, Erlangen, Germany) and PET/CT (Biograph mCT, Siemens Healthcare, Erlangen, Germany) before the start of RT. The studies were approved by the local ethics committee. All patients gave written informed consent for participating in the imaging studies.

For 7 patients (group A) the PET/MR imaging session was performed about $2 \mathrm{~h}(120-166 \mathrm{~min}$, median: $129 \mathrm{~min})$ after injection of FDG (320-388 MBq, median: $357 \mathrm{MBq})$. The other 8 patients (group B) were imaged 0-40 min after injection of FMISO (165-377 MBq, median: $339 \mathrm{MBq})$ in PET/CT using a dynamic acquisition mode, with a subsequent PET/MR imaging session about $3 \mathrm{~h}$ p.i. (164$206 \mathrm{~min}$, median: $174 \mathrm{~min})$. For these patients, an additional FDG-PET/CT (307-354 MBq, median: 330 MBq) acquired 
1 - 30 days earlier (median: 8 days) at about $1 \mathrm{~h}$ p.i. (55$81 \mathrm{~min}$, median: $71 \mathrm{~min}$ ) was also available. An overview of the patient cohort including the imaging data available for each patient is shown in Table 1.

PET images obtained from PET/MR were reconstructed to a voxel size of $2.8 \times 2.8 \times 2.0 \mathrm{~mm}^{3}$ using an OSEM $3 \mathrm{D}$ algorithm with 2 iterations and 21 subsets (2i21s) and a 3D Gaussian filter of $4 \mathrm{~mm}$. MR-based PET attenuation correction was performed by a vendor-provided segmentation approach based on spoiled gradient-echo sequences with DIXON-based fat-water separation [28]. FMISO-PET images from the PET/CT were reconstructed to a voxel size of $4.1 \times 4.1 \times 5.0 \mathrm{~mm}^{3}$ using OSEM 3D with $4 \mathrm{i} 8 \mathrm{~s}$ and a 3D Gaussian filter of $5 \mathrm{~mm}$. FDG-PET images from the $\mathrm{PET} / \mathrm{CT}$ were reconstructed to a voxel size of $2.0 \times 2.0 \times$ $3.0 \mathrm{~mm}^{3}$ using OSEM 3D with $3 \mathrm{i} 24 \mathrm{~s}$ and a $3 \mathrm{D}$ Gaussian filter of $3 \mathrm{~mm}$.

MRI acquisitions at the Biograph mMR were performed with the standard 16 channel head neck coil. An anatomical, transversal T2-weighted acquisition using a short time inversion recovery (STIR) sequence was acquired for each patient (repetition time (TR)/echo time (TE)/inversion time $(\mathrm{TI})=4830 \mathrm{~ms} / 37 \mathrm{~ms} / 220 \mathrm{~ms}$, flip angle $160^{\circ}$, voxel size $0.7 \times 0.7 \times 4.8 \mathrm{~mm}^{3}$, bandwidth $220 \mathrm{~Hz} / \mathrm{px}, 2$ averages, acquisition time $3 \mathrm{~m} 58 \mathrm{~s}$ ).

In addition, DW-MR images were obtained using a single-shot spin-echo echo-planar imaging (TR/TE $=7400 \mathrm{~ms} / 49 \mathrm{~ms}, \quad b$-values $50 \mathrm{~s} / \mathrm{mm}^{2}$ and $800 \mathrm{~s} / \mathrm{mm}^{2}$, bandwidth $2083 \mathrm{~Hz} / \mathrm{px}$, voxel size $2.1 \times 2.1 \times 5.0 \mathrm{~mm}^{3}, 3$ averages, spectral attenuated inversion recovery fat suppression, acquisition time $2 \mathrm{~m} 26 \mathrm{~s})$. ADC maps were obtained from the scanner software (Syngo MR B18P).

For $N=5$ patients also DCE-MR datasets were obtained. An axial T1-weighted fast spoiled gradient echo sequence (TWIST, TR/TE $=2.86 \mathrm{~ms} / 1.01 \mathrm{~ms}$, flip angle $12^{\circ}$, voxel size $1.1 \times 1.1 \times 4.0 \mathrm{~mm}^{3}$, temporal resolution $2.9 \mathrm{~s}$, bandwidth $530 \mathrm{~Hz} / \mathrm{px}$, acquisition time $4 \mathrm{~m} 18 \mathrm{~s}$ ) was performed after an automatic fast bolus injection of $0.1 \mathrm{mmol} \mathrm{Gd}$ DTPA per kg patient weight, followed by a saline flush. The field of view included the entire tumor and the common carotid arteries.

For the derivation of the native longitudinal relaxation times needed for DCE-quantification, additional VIBE sequences were acquired with two different flip angles $\left(\alpha_{1}=2^{\circ}, \alpha_{2}=12^{\circ}\right)$ before contrast agent injection $(\mathrm{TR} / \mathrm{TE}=4.04 \mathrm{~ms} / 1.52 \mathrm{~ms})$. The image grid was identical to the one of the DCE-MR acquisitions.

\section{Calculation of parameter maps}

The activity of the dynamic FMISO-PET datasets acquired during tracer wash-in was integrated for each voxel between 0 to 4 min p.i. using the rectangle method. By normalizing with respect to the acquisition time range of $4 \mathrm{~min}$, a map of mean activity, $\bar{A}_{\text {FMISO }}$, was obtained. For DCE images, the time-dependent signal enhancement of each voxel was

Table 1 Patient characteristics and acquired datasets

\begin{tabular}{|c|c|c|c|c|c|c|c|c|c|}
\hline \multirow[b]{2}{*}{ Patient } & \multirow[b]{2}{*}{ Gender } & \multirow[b]{2}{*}{ Age } & \multirow{2}{*}{$\begin{array}{l}\text { Tumor } \\
\text { localization }\end{array}$} & \multirow{2}{*}{$\begin{array}{l}\text { volume of } \\
\text { GTV }\left[\mathrm{cm}^{3}\right]\end{array}$} & \multirow{2}{*}{$\begin{array}{l}\text { imaging } \\
\text { modalities }^{a}\end{array}$} & \multirow{2}{*}{$\begin{array}{l}\text { DCE- } \\
\mathrm{MRI}^{b}\end{array}$} & \multirow{2}{*}{$\begin{array}{l}\text { DW- } \\
\text { MRI }^{c}\end{array}$} & \multicolumn{2}{|l|}{ \# samples } \\
\hline & & & & & & & & voxel level & regional level \\
\hline 1 & $\mathrm{~m}$ & 70 & Hypopharynx & 35 & $\mathrm{~A}$ & - & $\mathrm{x}$ & 453 & $7^{+}$ \\
\hline 2 & $\mathrm{f}$ & 57 & Oropharynx & 41 & $\mathrm{~A}$ & - & $\mathrm{x}$ & 2519 & 24 \\
\hline 3 & $\mathrm{~m}$ & 62 & Larynx & 16 & $\mathrm{~A}$ & - & $\mathrm{x}$ & 1050 & 11 \\
\hline 4 & $\mathrm{~m}$ & 64 & Oropharynx & 19 & A & - & $\mathrm{x}$ & 1172 & 13 \\
\hline 5 & $\mathrm{~m}$ & 44 & Cervical lymph node & 19 & A & - & $\mathrm{x}$ & 1176 & 12 \\
\hline 6 & $\mathrm{f}$ & 77 & Nasopharynx & 16 & A & - & $\mathrm{x}$ & 1005 & 12 \\
\hline 7 & $\mathrm{~m}$ & 52 & Hypopharynx & 16 & A & - & $\mathrm{x}$ & 743 & $6^{+}$ \\
\hline 8 & $\mathrm{f}$ & 62 & Base of tongue & 16 & B & $\mathrm{x}$ & $(\mathrm{x})^{*}$ & 1008 & 13 \\
\hline 9 & $\mathrm{f}$ & 57 & Oropharynx & 23 & B & $\mathrm{x}$ & $\mathrm{x}$ & 423 & 21 \\
\hline 10 & $\mathrm{~m}$ & 48 & Oropharynx & 33 & B & $\mathrm{x}$ & $(\mathrm{x})^{*}$ & 2112 & 30 \\
\hline 11 & $\mathrm{~m}$ & 56 & Hypopharynx & 50 & B & $\mathrm{x}$ & $\mathrm{x}$ & 3148 & 45 \\
\hline 12 & $\mathrm{~m}$ & 64 & Oropharynx & 167 & B & - & $\mathrm{x}$ & 10462 & 166 \\
\hline 13 & $\mathrm{~m}$ & 57 & Oropharynx & 10 & B & - & $\mathrm{x}$ & 638 & $6^{+}$ \\
\hline 14 & $\mathrm{f}$ & 55 & Oropharynx & 56 & B & $\mathrm{x}$ & $\mathrm{x}$ & 3556 & 52 \\
\hline 15 & $\mathrm{f}$ & 53 & Oropharynx & 32 & $\mathrm{~B}$ & - & $\mathrm{x}$ & 2005 & 27 \\
\hline
\end{tabular}

${ }^{a}$ A: FDG-PET/MR $2 \mathrm{~h}$ p.i., B: FDG-PET/CT $1 \mathrm{~h}$ p.i. + dynamic FMISO-PET/CT 0-40 min p.i. + FMISO-PET/MR 3 h p.i., ${ }^{b} \mathrm{x}:$ DCE-MRI acquired, -: no DCE-MRI available, ${ }^{c} \mathrm{x}$ : ADC-map acquired and evaluated, (x)* ADC-map omitted due to spatial distortions (according to visual assessment), ${ }^{+}$not used for evaluation due to small sample size 
calculated by subtraction of the mean signal before contrast agent injection. Subsequently, maps of mean signal enhancement $\overline{\Delta S}_{\text {DCE }}$ from 0 to 4 min p.i. were calculated analogous to the $\bar{A}_{\text {FMISO }}$ maps.

Before compartmental analysis, DCE images were resampled to the FMISO image grid from PET/MR. Signaltime-curves of DCE were fitted with an in-house implemented software (Matlab R2014b) using the extended Tofts model [29] and the Levenberg-Marquardt least squares algorithm. The arterial input function (AIF) was derived for each patient independently from a fit of the signal-time curve in the common carotid artery. Parameter maps of the volume fraction of the extracellular-extravascular space (EES), $v_{\mathrm{e}}$, the volume fraction of the blood plasma, $v_{\mathrm{p}}$, and the volume transfer rate from plasma to the EES, $K^{\text {trans }}$, were obtained. For regional analysis (see below) compartmental analysis was performed separately on the regional level of $3 \times 3 \times 4$ voxels of the resampled DCE images.

\section{Image registration and tumor volume delineation}

For performing the correlation analysis, the GTV of each patient from group A was delineated by an experienced radiation oncologist based on combined information of the FDG-PET and the T2-weighted STIR image [30]. ADC maps were resampled to match the FDG image grid with b-spline interpolation of 3rd order using the Insight Segmentation and Registration Toolkit (ITK version 4.5.2, www.itk.org).

For delineation of the GTVs of group B, manual contours defined by an experienced radiation oncologist on corresponding planning CTs were transferred to the PET/MR datasets by deformable registration of the CT and STIR images. The registrations were performed with elastix [31] using a b-spline parametrized transform and mutual information as similarity measure. Details of the used deformable registration algorithm and the respective parameter set are given in [32]. ADC images from PET/MR were resampled to match the FMISO image grid. Additionally, the FDG image from PET/CT was transformed to the FMISO image grid from PET/MR by deformable registration of the corresponding CT and STIR images with the method described above. The $\bar{A}_{\text {FMISO }}$ map was registered to the PET/MR dataset in the same way.

\section{Statistical analysis}

Correlation analysis was performed for all available pairwise combinations of functional and parametric maps with Python 2.7.6 using the SciPy library (www.scipy.org). Correlations were quantified by evaluating for each patient seperately the Spearman correlation coefficient on a voxel as well as on a regional level within the GTV. For voxelbased analysis, samples were defined by the PET image grid from PET/MR. For regional analysis, samples were defined as averages from non-overlapping sub-regions of the GTV. Each sub-region was defined over $3 \times 3 \times 4$ voxels of the PET image grid, corresponding to a size of $8.4 \times 8.4 \times 8 \mathrm{~mm}^{3}$. Patients with less than ten subregions were excluded from regional analysis (cf. Table 1).

\section{Results}

All functional images and parameter maps used for pairwise correlation analysis, together with the anatomical STIR acquisition and the delineated GTV, are exemplarily visualized for Patient 11 in Fig. 1.

Exemplary scatter plots of the voxel-based pairwise correlation analysis are shown in Fig. 2, visualizing results of two exemplary patients. Scatter plots and corresponding correlation coefficients show that there were patients for which pairs of functional data which showed rather strong correlations (e.g. FDG/ADC, $\overline{\Delta S}_{\mathrm{DCE}} / \bar{A}_{\mathrm{FMISO}}$ for Patient 11 ), while for other patients the correlations of the same pairs were much lower (cf. Patient 14).

Figure 3 shows a correlation matrix representing the median Spearman correlation coefficients obtained over the available patient datasets for all pairwise combinations of functional data, both for voxel-based and regional analysis. Highest inter-modality median coefficients of the voxel-based analysis were obtained for the combinations FDG/FMISO ( $r=0.56$, range: $0.08-0.80, N=8$ ), FDG $/ \bar{A}_{\text {FMISO }}(r=0.55$, range: $0.19-0.76, N=8)$, $\bar{A}_{\mathrm{FMISO}} / \overline{\Delta S}_{\mathrm{DCE}}(r=0.46$, range: $0.30-0.57, N=5)$, and ADC/FDG ( $r=-0.39$, range: $-0.82-0.30, N=13)$. For regional analysis, values changed to FDG/FMISO $(r=$ 0.51 , range: $0.06-0.86, N=7), \mathrm{FDG} / \bar{A}_{\mathrm{FMISO}}(r=0.32$, range: $-0.02-0.61, N=7), \bar{A}_{\mathrm{FMISO}} / \overline{\Delta S}_{\mathrm{DCE}}(r=0.40$, range: $-0.09-0.61, N=5)$, and $\mathrm{ADC} / \mathrm{FDG}(r=-0.28$, range: $-0.98-0.62, N=10$ ).

The inter-patient variation of Spearman correlation coefficients for both voxel and regional analysis are shown in Figure 4 for the pairs of highest median voxel correlations. Moreover, correlation coefficients are shown for each patient individually in Fig. 5.

\section{Discussion}

While weak correlations were observed between some functional imaging modalities in the median over all patient datasets, distinct correlations were only present on an individual basis. This applies both to the voxel-based and the 
Fig. 1 Dataset of Patient 11, showing transversal slices of the anatomical T2-weighted image (STIR), ADC map, FMISO image at $3 \mathrm{~h}$ p.i., the DCE-derived maps $K^{\text {trans }}, v_{\mathrm{e}}$ and $v_{\mathrm{p}}$, the maps of mean signal enhancement $\overline{\Delta S}_{\mathrm{DCE}}$ and mean FMISO activity $\bar{A}_{\text {FMISO }}$ in the time range of $0-4 \mathrm{~min}$ p.i, and the FDG image at $1 \mathrm{~h}$ p.i. All images and parameter maps were acquired in a single PET/MRI session, except for the FDG image and the $\bar{A}_{\text {FMISO }}$ map which were transferred to the PET/MR dataset by deformable registration. The delineation of the GTV is shown in red
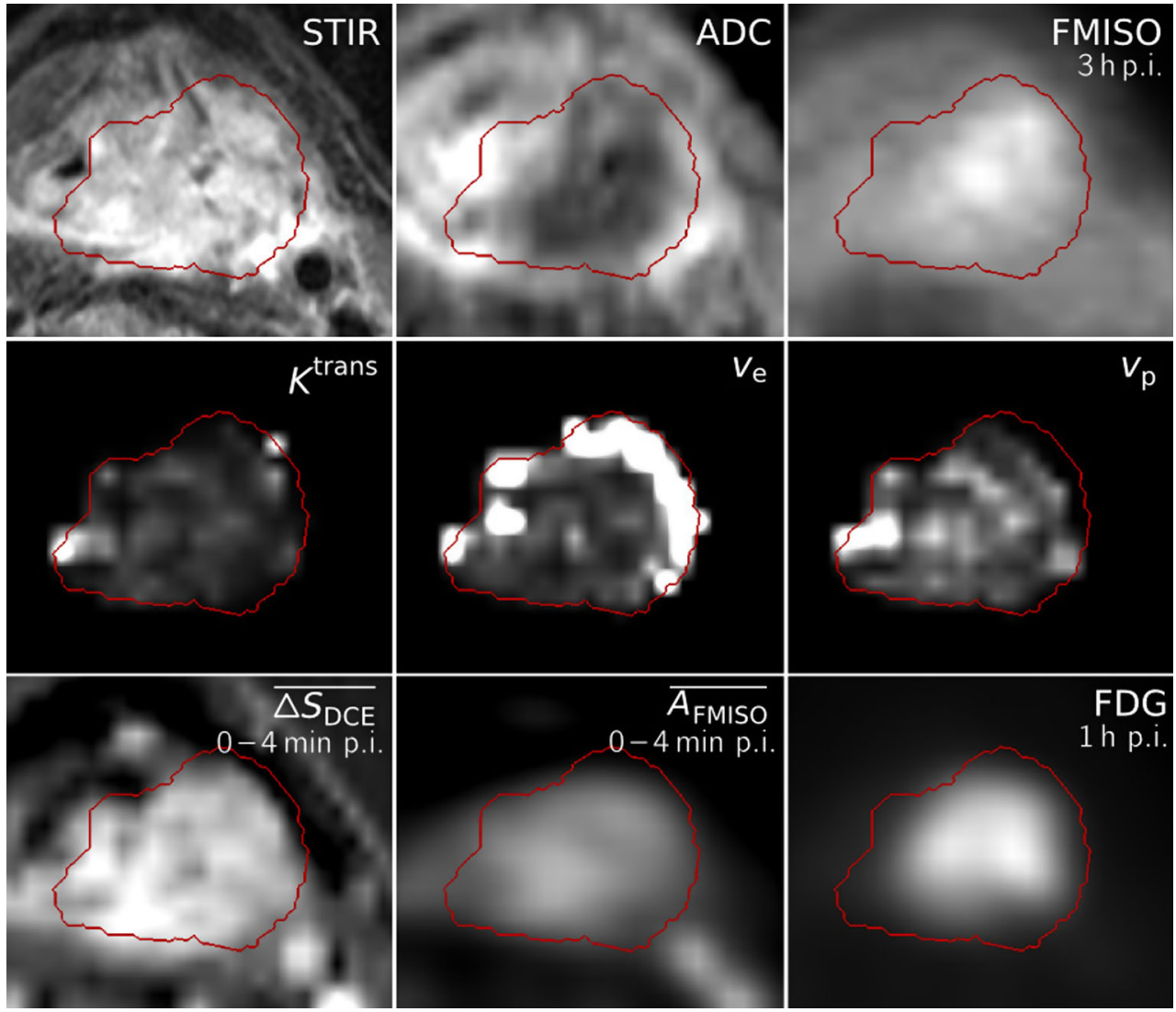

regional analysis. FDG and FMISO showed the largest intermodality median correlations in our study. However, the respective correlation coefficients varied strongly within the patient cohort. This result is in line with the findings of Rajendran et al. [20] and Thorwarth et al. [21]. Similar to Houweling et al. [23], moderate negative correlations were observed between FDG and ADC, with a pronounced variability across patients. No correlations were observed between FMISO and DCE-derived parameters maps. This is different from Newbold et al. [24], Donaldson et al. [25], and Jansen et al. [26]. However, our study is not readily comparable to the results of these authors, since the first authors quantified hypoxia by means of pimonidazole staining after tumor resection and the latter performed the analysis for neck nodal metastases.

We also found a moderate correlation between $\bar{A}_{\text {FMISO }}$ and $\overline{\Delta S}_{\mathrm{DCE}}$. This indicates that they may be measures of similar physiological parameters. However, according to this first analysis the observed correlation does not seem to be sufficient to infer the early FMISO information during wash-in from the DCE data. Instead of using $\overline{\Delta S}_{\text {DCE }}$ maps directly for correlation analysis, they could have also been converted to maps of contrast agent concentration using native T1 maps derived from the VIBE acquisitions. While this might have a slight impact on correlations quantified with the Spearman coefficient due to the dependency of the relation between signal enhancement and concentration on native T1, the conversion to concentration maps would introduce an additional source of error due to uncertainties in native $\mathrm{T} 1$ derivation.

For ADC and $v_{\mathrm{e}}$ maps, correlations may be expected as ADC is commonly related to the fraction of EES, and $v_{\mathrm{e}}$ is interpreted as the fraction of EES itself. However, in our study weak correlations are only observed on a regional level. One explanation of missing correlations could be that DCE parameter maps in regions with low vascularization are not reliable due to the weak delivery of contrast agent. However, correlation analysis between ADC and $v_{\mathrm{e}}$ should be performed with further datasets to provide more representative results.

Some of the highest correlations were found between the DCE-based maps $K^{\text {trans }}, v_{\mathrm{e}}, v_{\mathrm{p}}$, as well as $\overline{\Delta S}_{\mathrm{DCE}}$. This may be either due to inherently correlated parameter estimates in the extended Tofts model used for data analysis, or due to biological relations between the respective parameters.

The determination of multimodal parameter correlations may be substantially compromised by different factors, such as geometrical inaccuracies associated with imaging techniques and image registration, as well as interpolation errors and statistical uncertainties. Geometrical 

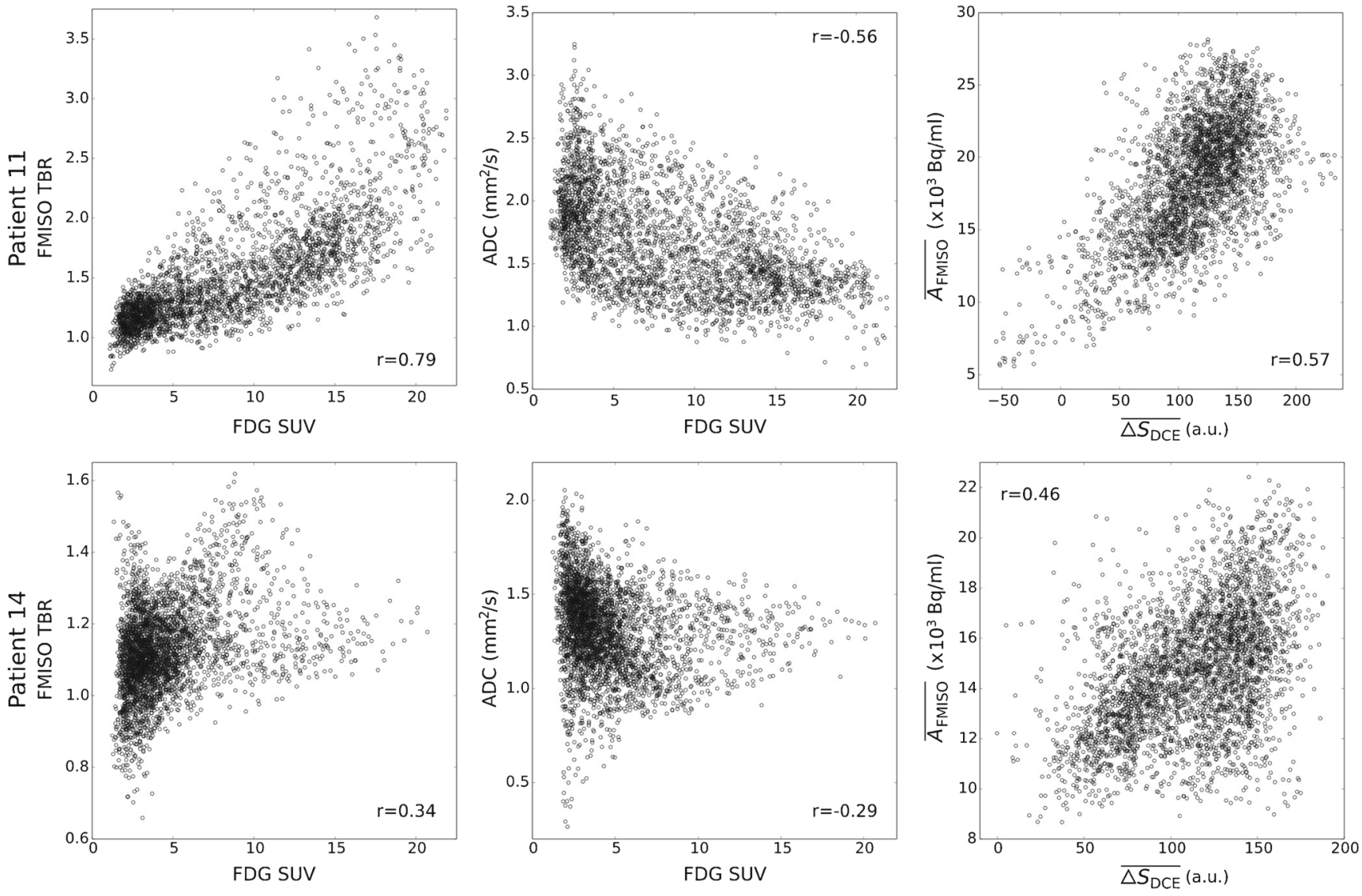

Fig. 2 Exemplary scatter plots for Patients 11 (top) and 14 (bottom), with samples obtained on the voxel level. For increased compara-

devision by the background signal in a deep neck muscle, resulting bility across patients, FDG activity concentrations were converted to standardized uptake values (SUVs). FMISO data was normalized by in the tumor to background ratio (TBR). The Spearman correlation coefficients $r$ associated with the scatterplots are shown within each plot

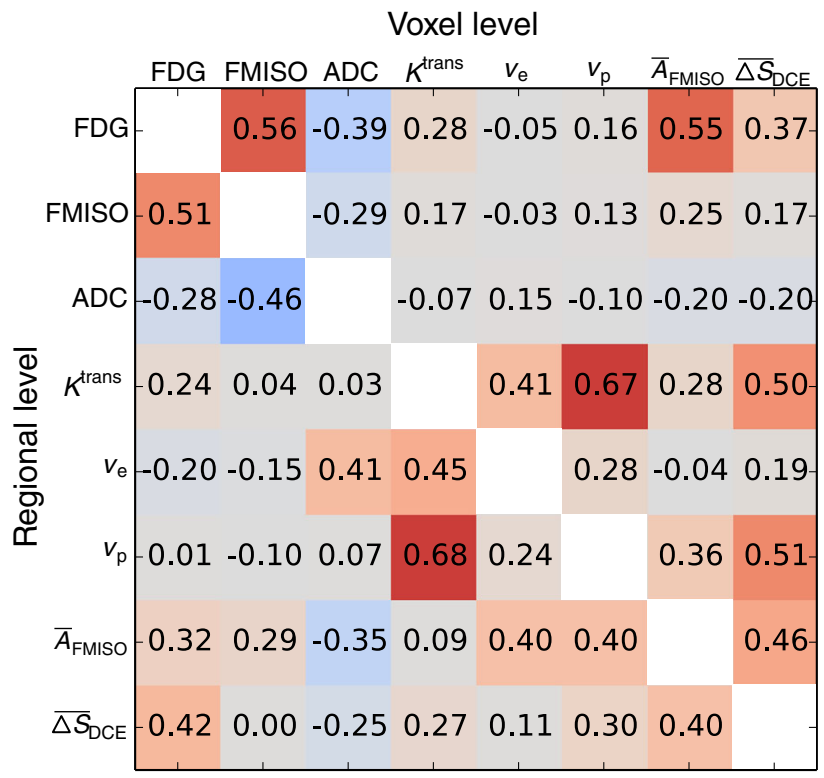

Fig. 3 Correlation matrix showing for each pair of functional information the median Spearman correlation coefficients obtained over all respective patient datasets. The upper right triangle shows coefficients derived on the voxel level, whereas the lower left triangle shows the coefficients derived on the regional level 


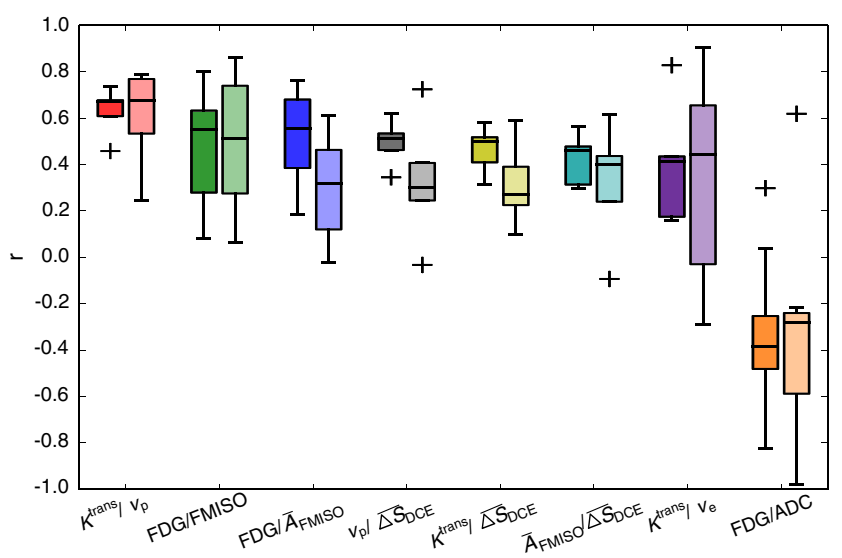

Fig. 4 Boxplots showing the inter-patient variation of Spearman correlation coefficients for the pairs with the median voxel correlations according to Fig. 3. For each functional pair, the results from voxel-based analysis (dark color, left boxes) and from regional analysis (light color, right boxes) are shown

distortions are particularly present in the ADC maps, which were acquired using EPI sequences. For future acquisitions, ideally sequences which are less prone to geometrical distortions should be used in combination with a method for geometrical distortion correction [33, 34]. Also, since no patient positioning system was used during image acquisition in the combined PET/MR examinations, movement of the patients during image acquisition cannot be excluded a priori. Hardware solutions for effective patient immobilization are currently being developed [35]. Finally, geometrical uncertainties are associated with images that were transferred to the PET/MR datasets by deformable registration, which may lead to a reduction of absolute correlation values [36]. An independent analysis of the errors introduced by the different factors is not possible with realistic patient data. In order to account for geometrical uncertainties, a correlation analysis on a regional level was added to the voxel analysis. Such a regional analysis is more robust with respect to geometrical uncertainties, interpolation errors and image noise, whereas averaging may underestimate existing correlations, and additional statistical uncertainties may be introduced. Both increases and decreases in correlation coefficients compared to the voxel-based analysis were observed. However, similar inter-patient distributions were observed (cf. Fig. 4). As a main result of our study we found large variations of correlation coefficients between patients, which most probably can not be explained by the present inaccuracies alone.

DCE parameter maps were derived with the extended Tofts model. However, model parameters may be misinterpreted for some physiological conditions such as highly vascularized tissues with intermediate flow [37]. For other conditions, the model may not fit the data accurately. Other models with fewer assumptions like the four parameter

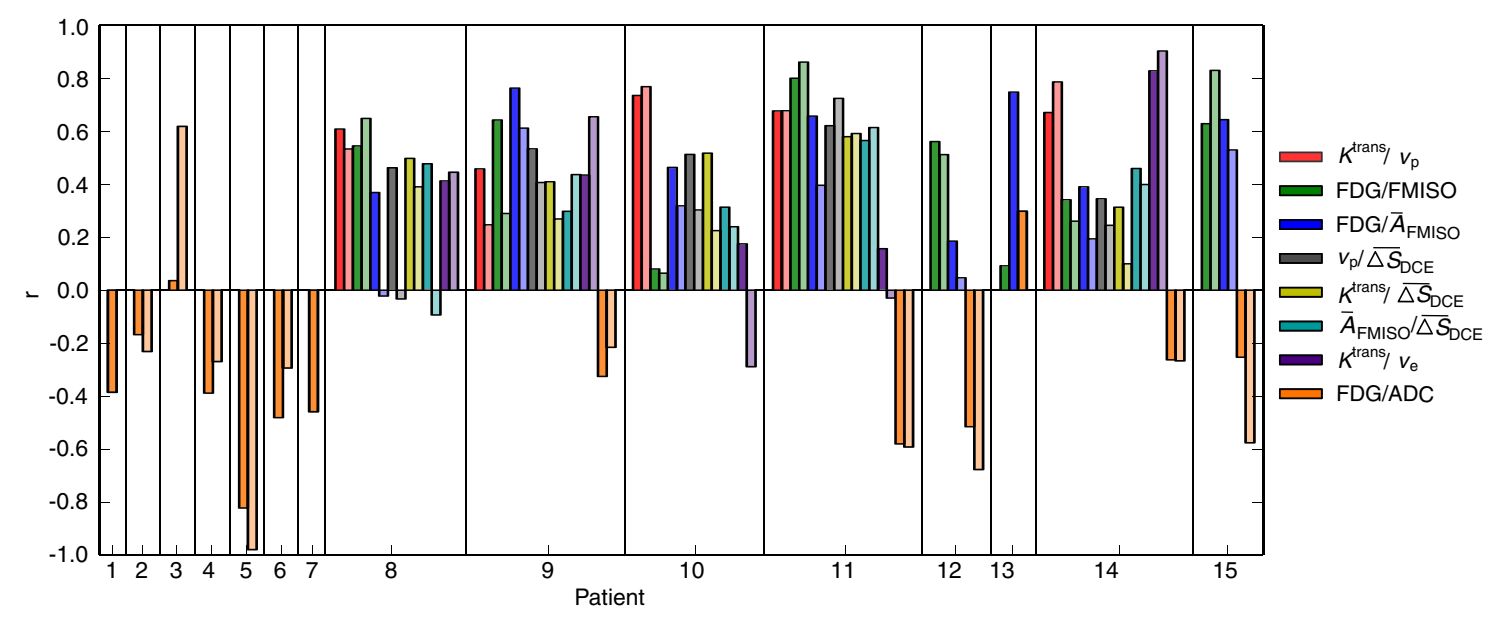

Fig. 5 Spearman correlation coefficients obtained for the individual patients. Color encoding is the same as in Fig. 4, with dark and light colors showing the results from voxel-based and regional analysis, respectively 
two-compartment exchange model (2CXM) could be used instead if data quality is sufficient in terms of temporal resolution, signal-to-noise ratio and artifacts [6].

Only a limited number of patients was available in this study, especially with respect to DCE-MRI data. Further evaluation should be performed when more patient data is available.

The results in this study extend the correlation analyses performed in previous studies by considering a comprehensive set of functional data. The present results suggest that the different functional datasets derived from DCE-MRI, DW-MRI, FDG-PET and FMISO-PET provide complementary information. Since all these imaging methods were proven to be prognostic for treatment outcome [11-19], this suggests that each method may be of separate value for the adaptation of treatment strategies. However, only pairwise correlations have been analyzed so far. It appears interesting to elaborate if the information from one functional imaging method could be deduced from a combination of several other functional imaging methods. Such an analysis could in the future be performed with machine learning approaches [38] when more patient datasets are available. On the other hand, one may obtain more coherent correlation results if only subgroups of HN tumors are analyzed, for example patients with equal tumor localization, size and staging.

Analyses exploring a potential redundancy between functional PET/MR data may be of value for RT and other treatment modalities due to several reasons. Firstly, using redundant image data and parameter maps for biologically adapted treatments would unnecessarily increase the number of parameters to be adapted with respect to improved outcome. Thus, the correlation analysis performed in the present study constitutes a first step towards the integration of functional imaging into treatment individualization. Before biologically adapted treatments can be used clinically, a number of additional steps are required, such as the correlation of functional parameters to treatment outcome and a thorough regional failure analysis. Further research is needed to clarify which parameter combination provides accurate information about locoregional control probability. Secondly, functional imaging data may concatenate multiple physiological parameters, and interpretation is not always straightforward. A more detailed understanding of functional images and the parameter maps obtained by post-processing models is necessary [37, 39]. Exploring a potential inter-dependency between different datasets may support the interpretation of functional imaging data. Moreover, present or missing correlations between different datasets could potentially also be associated with biological evidence related to treatment response of individual patients. A more comprehensive picture of these issues would allow for a knowledge-driven treatment adaptation, which would then need to be validated in clinical trials.

\section{Conclusion}

Multiparameteric PET/MR provides a substantial amount of different functional imaging data, which may be highly beneficial for cancer treatment adaptation. The results of our study suggest that the associated datasets provide complementary information, and thus could all be of separate value for defining treatment adaption strategies, as well as for treatment response assessment and followup. Results of this correlation study might in the future contribute to the design of individually adapted treatment approaches based on multiparametric functional PET/MR imaging.

\section{Compliance with Ethical Standards}

Funding Parts of this project were supported by the European Research Council (ERC), starting Grant No. 335367.

\section{Conflict of interests None.}

Ethical approval All procedures performed in studies involving human participants were in accordance with the ethical standards of the institutional research committee and with the 1964 Helsinki declaration and its later amendments or comparable ethical standards.

Informed consent Informed consent was obtained from all individual participants included in the study.

Open Access This article is distributed under the terms of the Creative Commons Attribution 4.0 International License (http://creativecommons.org/licenses/by/4.0/), which permits unrestricted use, distribution, and reproduction in any medium, provided you give appropriate credit to the original author(s) and the source, provide a link to the Creative Commons license, and indicate if changes were made.

\section{References}

1. Dirix P, Vandecaveye V, De Keyzer F, Stroobants S, Hermans R, Nuyts S. Dose Painting in Radiotherapy for Head and Neck Squamous Cell Carcinoma: Value of Repeated Functional Imaging with 18F-FDG PET, 18F-Fluoromisonidazole PET, DiffusionWeighted MRI, and Dynamic Contrast-Enhanced MRI. J Nucl Med. 2009;50(7):1020-1027.

2. Subesinghe M, Scarsbrook AF, Sourbron S, Wilson DJ, McDermott G, Speight R, et al. Alterations in anatomic and functional imaging parameters with repeated FDG PET-CT and MRI during radiotherapy for head and neck cancer: a pilot study. BMC cancer. 2015;15:137.

3. Overgaard J, Eriksen JG, Nordsmark M, Alsner J, Horsman MR. Plasma osteopontin, hypoxia, and response to the hypoxia sensitiser nimorazole in radiotherapy of head and neck cancer: results from the DAHANCA 5 randomised double-blind placebocontrolled trial. Lancet Oncol. 2005;6(10):757-764.

4. Carmeliet P, Jain RK. Principles and mechanisms of vessel normalization for cancer and other angiogenic diseases. Nat Rev Drug Discov. 2011;10(6):417-427. 
5. Padhani AR, Liu G, Mu-Koh D, Chenevert TL, Thoeny HC, Takahara T, et al. Diffusion-Weighted Magnetic Resonance Imaging as a Cancer Biomarker: Consensus and Recommendations. Neoplasia. 2009;11(2):102-125.

6. Donaldson SB, West CML, Davidson SE, Carrington BM, Hutchison G, Jones AP, et al. A comparison of tracer kinetic models for T1-weighted dynamic contrast-enhanced MRI: Application in carcinoma of the cervix. Magn Reson Med. 2010;63(3):691-700.

7. Kolb A, Wehrl H, Hofmann M, Judenhofer M, Eriksson L, Ladebeck R, et al. Technical performance evaluation of a human brain PET/MRI system. Eur Radiol. 2012;22(8):1776-1788.

8. Delso G, Fürst S, Jakoby B, Ladebeck R, Ganter C, Nekolla SG, et al. Performance measurements of the Siemens mMR integrated whole-body PET/MR scanner. J Nucl Med. 2011;52(12):19141922.

9. Thorwarth D, Leibfarth S, Mönnich D. Potential role of PET/MRI in radiotherapy treatment planning. Clin Transl Imaging. 2013;1:45-51.

10. Yankeelov TE, Peterson TE, Abramson RG, Garcia-Izquierdo D, Arlinghaus LR, Li X, et al. Simultaneous PET-MRI in oncology: a solution looking for a problem? Magn Reson Imaging. 2012;30(9):1342-1356.

11. Pak K, Cheon GJ, Nam HY, Kim SJ, Kang KW, Chung JK, et al. Prognostic Value of Metabolic Tumor Volume and Total Lesion Glycolysis in Head and Neck Cancer: A Systematic Review and Meta-Analysis. J Nucl Med. 2014;55(6):884-890.

12. Koyasu S, Nakamoto Y, Kikuchi M, Suzuki K, Hayashida $\mathrm{K}$, Itoh K, et al. Prognostic Value of Pretreatment 18F-FDG PET/CT Parameters Including Visual Evaluation in Patients With Head and Neck Squamous Cell Carcinoma. Am J Roentgenol. 2014;202(4):851-858.

13. Rischin D, Hicks RJ, Fisher R, Binns D, Corry J, Porceddu $\mathrm{S}$, et al. Prognostic significance of [18F]-misonidazole positron emission tomography-detected tumor hypoxia in patients with advanced head and neck cancer randomly assigned to chemoradiation with or without tirapazamine: A substudy of TransTasman Radiation Oncology Group Study 98.02. J Clin Oncol. 2006;24(13):2098-2104.

14. Mortensen LS, Johansen J, Kallehauge J, Primdahl H, Busk M, Lassen P, et al. FAZA PET/CT hypoxia imaging in patients with squamous cell carcinoma of the head and neck treated with radiotherapy: Results from the DAHANCA 24 trial. Radiother Oncol. 2012;105:14-20.

15. Zips D, Zöphel K, Abolmaali N, Perrin R, Abramyuk A, Haase R, et al. Exploratory prospective trial of hypoxia-specific PET imaging during radiochemotherapy in patients with locally advanced head-and-neck cancer. Radiother Oncol. 2012;105:2128.

16. Thorwarth D, Eschmann SM, Scheiderbauer J, Paulsen F, Alber M. Kinetic analysis of dynamic $18 \mathrm{~F}$-fluoromisonidazole PET correlates with radiation treatment outcome in head-and-neck cancer. BMC Cancer. 2005;5:152.

17. Lambrecht M, Calster BV, Vandecaveye V, Keyzer FD, Roebben I, Hermans R, et al. Integrating pretreatment diffusion weighted MRI into a multivariable prognostic model for head and neck squamous cell carcinoma. Radiother Oncol. 2014;110(3):429-434.

18. Andersen EK, Hole KH, Lund KV, Sundfør K, Kristensen GB, Lyng $\mathrm{H}$, et al. Pharmacokinetic parameters derived from dynamic contrast enhanced MRI of cervical cancers predict chemoradiotherapy outcome. Radiother Oncol. 2013;107:117-122.

19. Halle C, Andersen E, Lando M, Aarnes EK, Hasvold G, Holden $\mathrm{M}$, et al. Hypoxia-Induced Gene Expression in Chemoradioresistant Cervical Cancer Revealed by Dynamic Contrast-Enhanced MRI. Cancer Res. 2012;72(20):5285-5295.
20. Rajendran JG, Mankoff DA, O'Sullivan F, Peterson LM, Schwartz DL, Conrad EU, et al. Hypoxia and Glucose Metabolism in Malignant Tumors: Evaluation by [18F]Fluoromisonidazole and [18F]Fluorodeoxyglucose Positron Emission Tomography Imaging. Clin Cancer Res. 2004;10(7):2245-2252.

21. Thorwarth D, Eschmann SM, Holzner F, Paulsen F, Alber M. Combined uptake of [18F]FDG and [18F]FMISO correlates with radiation therapy outcome in head-and-neck cancer patients. Radiother Oncol. 2006;80(2):151-156.

22. Zegers CM, van Elmpt W, Reymen B, Even AJ, Troost EG, Öllers MC, et al. In Vivo Quantification of Hypoxic and Metabolic Status of NSCLC Tumors Using [18F] HX4 and [18F] FDG-PET/CT Imaging. Clin Cancer Res. 2014;20(24):63896397.

23. Houweling AC, Wolf AL, Vogel WV, Hamming-Vrieze O, van Vliet-Vroegindeweij C, van de Kamer JB, et al. FDG-PET and diffusion-weighted MRI in head-and-neck cancer patients: Implications for dose painting. Radiother Oncol. 2013;106(2):250 254.

24. Newbold K, Castellano I, Charles-Edwards E, Mears D, Sohaib A, Leach M, et al. An Exploratory Study Into the Role of Dynamic Contrast-Enhanced Magnetic Resonance Imaging or Perfusion Computed Tomography for Detection of Intratumoral Hypoxia in Head-and-Neck Cancer. Int J Radiat Oncol Biol Phys. 2009;74:29-37.

25. Donaldson SB, Betts G, Bonington SC, Homer JJ, Slevin NJ, Kershaw LE, et al. Buckley DL: Perfusion Estimated With Rapid Dynamic Contrast-Enhanced Magnetic Resonance Imaging Correlates Inversely With Vascular Endothelial Growth Factor Expression and Pimonidazole Staining in Head-and-Neck Cancer: A Pilot Study. Int J Radiat Oncol Biol Phys. 2011;81(4):11761183.

26. Jansen JF, Schöder H, Lee NY, Wang Y, Pfister DG, Fury MG, et al. Noninvasive Assessment of Tumor Microenvironment Using Dynamic Contrast-Enhanced Magnetic Resonance Imaging and 18F-Fluoromisonidazole Positron Emission Tomography Imaging in Neck Nodal Metastases. Int J Radiat Oncol Biol Phys. 2010;77(5):1403-1410.

27. Mönnich D, Troost EGC, Kaanders JHAM, Oyen WJG, Alber M, Zips D, et al. Correlation between tumor oxygenation and 18F-fluoromisonidazole PET data simulated based on microvessel images. Acta Oncol. 2013;52(7):1308-1313.

28. Martinez-Möller A, Souvatzoglou M, Delso G, Bundschuh RA, Chefd'hotel C, Ziegler SI, et al. Tissue Classification as a Potential Approach for Attenuation Correction in WholeBody PET/MRI: Evaluation with PET/CT Data. J Nucl Med. 2009;50(4):520-526.

29. Tofts P. Modeling tracer kinetics in dynamic Gd-DTPA MR imaging. J Magn Reson Imaging. 1997;7:91-101.

30. Leibfarth S, Eckert F, Welz S, Siegel C, Schmidt H, Schwenzer N, et al. Automatic delineation of tumor volumes by co-segmentation of combined PET/MR data. Phys Med Biol. 2015;60(14):5399.

31. Klein S, Staring M, Murphy K, Viergever M, Pluim J. elastix: A Toolbox for Intensity-Based Medical Image Registration. IEEE Trans Med Imaging. 2010;29:196-205.

32. Leibfarth S, Mönnich D, Welz S, Siegel C, Schwenzer N, Schmidt $\mathrm{H}$, et al. A strategy for multimodal deformable image registration to integrate $\mathrm{PET} / \mathrm{MR}$ into radiotherapy treatment planning. Acta Oncol. 2013;52(7):1353-1359.

33. Koyasu S, Iima M, Umeoka S, Morisawa N, Porter DA, Ito $\mathrm{J}$, et al. The clinical utility of reduced-distortion readoutsegmented echo-planar imaging in the head and neck region: initial experience. Eur Radiol. 2014;24(12):3088-3096. 
34. Windischberger C, Robinson S, Rauscher A, Barth M, Moser E. Robust field map generation using a triple-echo acquisition. J Magn Reson Imaging. 2004;20(4):730-734.

35. Paulus DH, Thorwarth D, Schmidt H, Quick HH. Towards integration of PET/MR hybrid imaging into radiation therapy treatment planning. Medical Physics. 2014;41(7):072505.

36. Nyflot MJ, Harari PM, Yip S, Perlman SB, Jeraj R. Correlation of PET images of metabolism, proliferation and hypoxia to characterize tumor phenotype in patients with cancer of the oropharynx. Radiother Oncol. 2012;105:36-40.
37. Sourbron SP, Buckley DL. On the scope and interpretation of the Tofts models for DCE-MRI. Magn Reson Med. 2011;66(3):735-745.

38. Ozer S, Langer DL, Liu X, Haider MA, van der Kwast TH, Evans AJ, et al. Supervised and unsupervised methods for prostate cancer segmentation with multispectral MRI. Med Phys. 2010;37(4):1873-1883.

39. Zwick S, Brix G, Tofts P, Strecker R, Kopp-Schneider A, Laue H, et al. Simulation-based comparison of two approaches frequently used for dynamic contrast-enhanced MRI. Eur Radiol. 2010;20(2):432-442. 